Voix et Images

\title{
Tout dépend de l'angle de la vision : par Nicole Brossard - La Lettre aérienne
}

\section{Patricia Smart}

Volume 11, numéro 2 (32), hiver 1986

Michel van Schendel

URI : https://id.erudit.org/iderudit/200564ar

DOI : https://doi.org/10.7202/200564ar

Aller au sommaire du numéro

Éditeur(s)

Université du Québec à Montréal

ISSN

0318-9201 (imprimé)

1705-933X (numérique)

Découvrir la revue

Citer cet article

Smart, P. (1986). Tout dépend de l'angle de la vision : par Nicole Brossard - La Lettre aérienne. Voix et Images, 11(2), 330-333. https://doi.org/10.7202/200564ar d'utilisation que vous pouvez consulter en ligne.

https://apropos.erudit.org/fr/usagers/politique-dutilisation/ 


\title{
Tout dépend de l'angle de la vision: par Nicole Brossard - La Lettre aérienne. ${ }^{1}$
}

\author{
par Patricia Smart, Université Carleton
}

La position occupée par Nicole Brossard comme chef de file de la «modernité» au Québec aura eu l'effet paradoxal d'éloigner d'elle certains lecteurs ou lectrices également rebutés par l'idée de faire partie d'une "chapelle» autour d'elle et par les attaques dont elle a été la cible (voir par exemple le dernier numéro de Voix et images, où son écriture est décrite comme un monument, une auvre type, déjà laissée en arrière par le "féminisme d'ici»!) ${ }^{2}$. Ce petit livre, pourtant très dense, arrive comme une invitation au voyage pour ceux et celles-là. Rassemblant des textes inédits écrits depuis 1975 et dont plusieurs ont été présentés à des colloques universitaires et/ou féministes, il témoigne de la même intensité, du même sentiment d'urgence, du même effort de logique et de communication qu'on remarque chez Nicole Brossard lorsqu'elle parle en public. On dirait que c'est quand elle s'astreint à la communication directe que le caractère visionnaire de la pensée de Brossard s'affirme le plus; comme si la vérité qu'elle entrevoit et qui l'anime insistait à ces moments-là pour être partagée avec un public non-initié. Urgence, amour, espoir animent cette lettre aérienne envoyée par Brossard de la spirale où elle tournoie à des destinataires encore emprisonnés dans les façons de penser du patriarcat. Et pourtant à chaque spire de la spirale elle revient à terre, à cette histoire en crise qui est celle de notre ère, ponctuée par les dernières répliques que la phallocratie adressera aux nouvelles valeurs qui prendront place (p. 11). Dès le premier texte du volume, écrit en 1975, c'est là qu'elle reconnaît son lieu d'enracinement: Je suis donc dans l'histoire jusqu'à la fin de mes jours (p. 11).

S'il est vrai que Brossard a été en quelque sorte transformée en institution, ces textes l'en sortent en révélant le courage d'un choix radical et à l'origine solitaire (celui du lesbianisme et du féminisme radical), assumé en entier et exploré sans compromission. De la première phrase du volume (Pour une fois, je ne veux pas parler des autres, ni au nom des autres, p. 11) jusqu'à la magnifique figure tridimensionnelle que Brossard appelle l'Intégrales (ni le féminin ni le pluriel ne sont fortuits) et qu'elle voit comme le but de son projet d'écriture et de vie, le trajet en est un de risque. Sortir du miroir patriarcal c'est devenir un point de mire - la cible, justement, des faiseurs de monuments et d'autres:

Je parle ici d'un certain angle de vision. Pour y arriver, il a fallu que je me déplace de manière à ce que le corps opaque du patriarcat n'empêche ma vision. Déplacée, je le suis, non pas comme une fille manquée mais comme celle que l'on manque, que l'on rate une fois qu'elle est mise en joue car la mire n'aura jamais les pouvoirs du miroir. Ce déplacement occasionne tous les autres. 
Deux textes d'avant 1980, «La plaque tournante» et "La coïncidence», se distinguent de ceux qui suivront par leur ton intime et tâtonnant, malgré la certitude de certaines constantes: l'histoire, le corps jouissif, l'importance de la solidarité entre femmes. L'image de la plaque tournante préfigure celle plus optimiste de la spirale qui sera élaborée au colloque «L'émergence d'une culture au féminin" à l'Université de Montréal en 1982. C'est l'île historique sur laquelle nous tournons tous dans un mouvement vertigineux qui approche de l'abîme, du centre blanc d'une période de transition. Île à laquelle les femmes, toutes en eau trouble (p. 13), s'accrochent mais où elles vivent solitaires, égarées, en exil. L'écriture de Brossard à cette époque en est une de fragments désirants à la recherche d'une continuité dans l'espace, d'un je accolé à la solitude par la force du moment historique: Je ne peux parler nous avant d'avoir su répliquer je (p. 13); D'être ici et maintenant une femme consciente et sans solidarité, ça n'a pas de sens (p. 21). Ensemble, ces deux premiers textes montrent les débuts d'un projet d'écriture signifiante situé dans une coïncidence avec d'autres femmes qui sera engendreuse de mouvement et de possibilités de sens: Si je désire une fermme, si une fermme me désire, c'est qu'il y a du commencement à l'écriture (p. 19). Cette période dans le trajet de Nicole Brossard apparaît tout de même comme celle de la traversée de l'abîme, d'une angoisse et d'une fatigue sans doute liées à la conscience d'une certaine stérilité dans l'expérimentation formelle de l'écriture de l'époque: Je suis fatiguée d'avoir un corps aux membres spécialisés, un corps-capital, un corps à histoires par personne interposée. Le textetrouble est sans doute fait de l'histoire de cette fatigue, allongée vive, dans le ghetto des arts... (p. 14). (Plus tard elle analysera avec plus de distance les limites de ce texte moderne qui par peur de ses origines parle le désir et non pas son désir (p. 45) et résume le corps au neutre-masculin, (p. 51.)

Il n'a jamais été question dans l'écriture de Brossard d'une prétendue "nature féminine" basée dans le corps et la nature: je ne puis m'imaginer confinée à la végétation, car le métal, le caillou, la cité m'entourent (p. 31). Il n'en reste pas moins que les femmes entre elles vivent et écrivent menacées, traversées par la peur, la colère et l'humiliation, asphyxiées dans l'espace opaque de la cité des pères (p.33). Veilleuses, bien en vue, dans la cité (p. 31), elles marchent sur une corde raide, confrontées au risque du vide, car nous travaillons sans filet (p. 33).

Ce qu'il y a de remarquable dans les autres textes du volume, c'est comment ils arrivent par un changement de l'angle de vision à retourner en affirmation cet espace de l'aliénation. Arpenté, touché, apprivoisé en la compagnie d'autres femmes, le vide devient le lieu où naît l'énergie (je dis qu'à même ma propre énergie, je sais le trou, sa texture, ses reliefs, son rythme, p. 39) et le centre blanc le point de départ d'une spirale dont le mouvement giratoire emmène en dehors du patriarcat tout en transformant l'espace historique dans lequel il prend son élan. Il suffit de regarder les mots et les choses autrement et tout peut arriver: en témoigne la merveilleuse analogie entre le parler-femmes et parler-avec-un-accent. Parlant la langue patriarcale avec un accent, et donc avec une intensité différente, les femmes 
peuvent se reconnaître et produire des transformations de sens inattendues, comme il arrive à ceux qui parlent une langue seconde.

La rencontre autour de 1980 des nouvelles théories de la physique contemporaine centrées sur l'hologramme (et de toute évidence celle de Mary Daly, de qui Brossard a publié un texte en traduction à l'époque) coïncide avec la trajectoire de Brossard dans le langage et semble l'ancrer davantage dans le réel. L'effort de perception exigé du lecteur par cette écriture est une invitation à entrer dans un "nouveau paradigme" qui dépasse et transcende la pensée binaire. Dense et toujours concret dans ses abstractions, le langage imagé de Brossard semble proche de la dialectique et du paradoxe. Mais il serait plus juste de l'appeler "post-dialectique» ou "post-paradoxal», car il part d'un point où il n'y a plus de contradiction dans l'idée d'une émotion de la pensée, d'une haute technologie sensuelle (p. 45) ou du poème qui me fait dire que les poitrines ont la raison du souffle que nous y trouvons, comme s'il s'agissait chaque fois d'écrire: je continue (p. 66). Tout dépend de l'angle de vision où l'on se situe et de la mobilité de l'approche.

Il a souvent été remarqué que la spirale de Brossard s'ouvre sur une dimension religieuse ou mystique de l'expérience, qui - grâce à l'intégration qu'elle fait des nouveaux questionnements en physique - se révèle au delà ou en dessous de nos représentations habituelles du réel. La racine est aérienne - la totalité se découvre à la racine des mots et du réel, dans le vertige d'une multiplication des perspectives:

C'est aussi pour chaque mot une rëvolution complète autour de son axe: on examine le radical sous tous ses angles, à tout point de vue. C'est l'excitation générale.

Lisant ces textes, on se demande si le mysticisme n'est au fond que l'aboutissement logique de notre désir et si c'est seulement avec l'avènement du féminisme qu'il a pu émerger comme un projet radical et compatible avec l'engagement dans l'histoire. Le contraste entre le projet de Brossard et celui d'Hubert Aquin, auquel il ressemble par plusieurs biais, me semble exemplaire à cet égard. Dans le cas d'Aquin, la fusion du mysticisme et de l'engagement accomplie dans Prochain épisode a été peu à peu minée par le dualisme d'une tradition patriarcale qui identifie l'objet du désir à la femme à posséder et à détruire: d'où, après la destruction apocalyptique de Neige noire, la paralysie et la disparition dans le «silence marginal» du texte. Chez Brossard, le regard d'une femme posé sur une autre femme n'a rien d'une possession ou d'un avalement; au contraire, il appelle à la traversée d'une "nouvelle dimension» et à une intervention dans la cité. L'Image de la lettre aérienne, dans laquelle on entre comme on entre dans sa peau (p. 64), désigne tout le projet d'écriture de Brossard comme une tentative de traduction d'un état second qui a toutes les caractéristiques d'une vision mystique - mais politique, précise, appelant à une transformation du réel:

Ainsi, au cour de la lettre aérienne, apparaissent clairement des zones difficilement repérables en temps normal quant à la vision politique que nous avons sur les êtres et les activités qu'ils prati- 
quent. C'est parce qu'elle ne fige jamais le regard que la vision aérienne donne à voir l'état de la réalité avec une incalculable précision. Quelque chose ici m'est proposé très précisément que je dois cependant découvrir en me mettant à l'œuvre, si je puis dire.

$\mathrm{Au}$ fond, la Lettre aérienne est un voyage initiatique dont on sort étourdi(e), mais extatique, il faut le dire. Une fois embarqué(es) sur la spirale de Nicole Brossard, on voit certaines choses comme une évidence. Ainsi, par exemple, son Intégrales, cette femme tridimensionnelle dans laquelle certains critiques ont vu un simple retour à la représentation traditionnelle, apparaît clairement, dans le vertige d'un regard qui apprend à laisser en arrière la division entre écriture et réalité, comme - tout simplement - cette femme réelle et imaginaire qui nous habite et qui ne demande qu'à être actualisée. Mais pour cela, il faut d'abord que j'invente ce que je suis (p. 115).

1. Montréal, Les Éditions du Remue-Ménage, 1985, 154 p.

2. Jean Fisette, «L'Écrevisse et l'impossible: gloses autour de deux textes de Nicole Brossard", Voix et images XI, no 1 (automne 1985), p. 75.

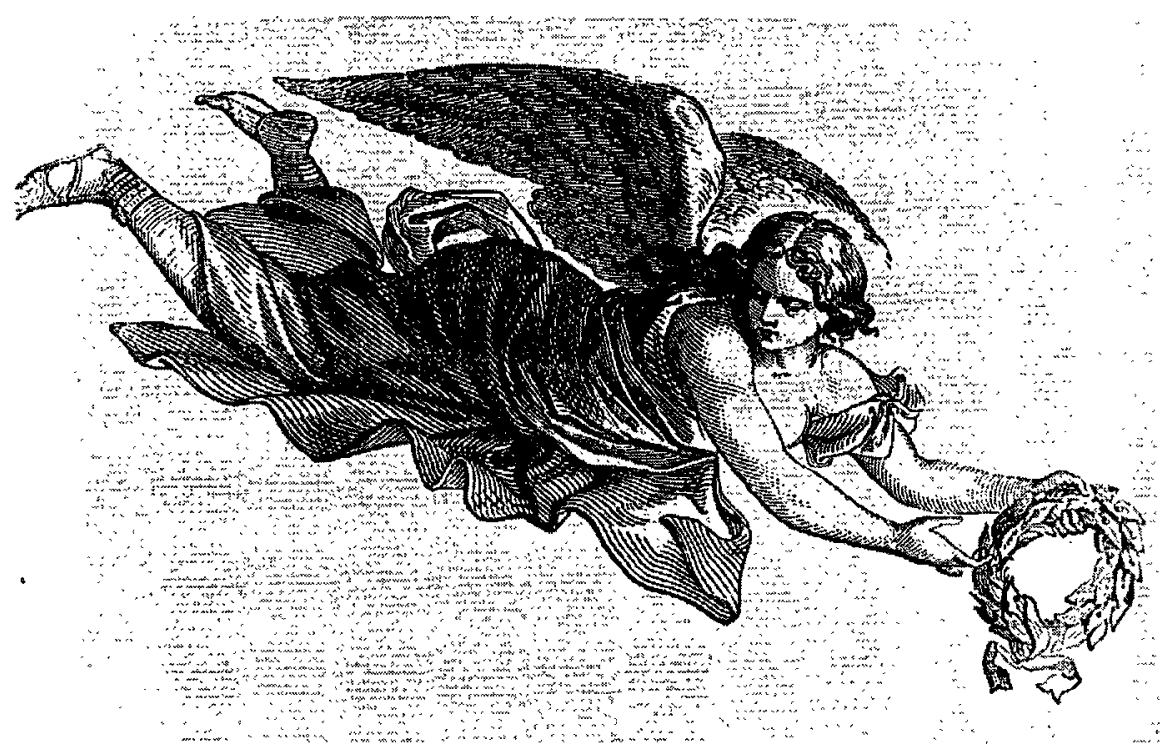

